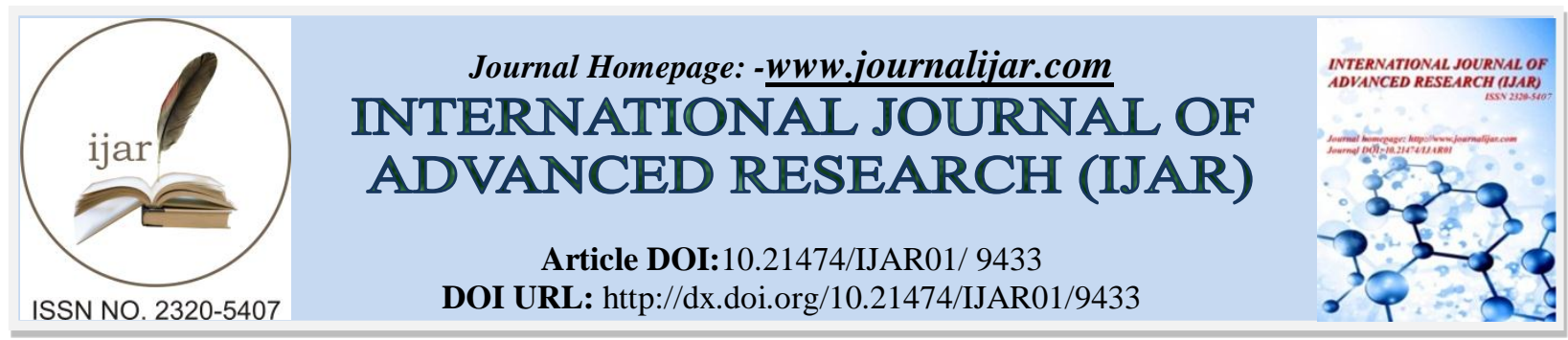

RESEARCH ARTICLE

\title{
DIABETES MELLITUS: CLASSIFICATION, EPIDEMIOLOGY, PHYSIOPATHOLOGY, IMMUNOLOGY, RISK FACTORS, PREVENTION AND NUTRITION.
}

\section{Fagninou Nonsito Adnette ${ }^{1}$, Tougan Polycarpe Ulbad ${ }^{2}$, Nekoua Magloire ${ }^{1}$, Fachina Ruffine ${ }^{1}$, Koutinhouin}

G.B. ${ }^{3}$ and Yessoufou Akadiri ${ }^{1}$.

1. Laboratory of Cell Biology and Physiology, Department of Biochemistry and Cellular Biology, Faculty of Sciences and Techniques, University of Abomey-Calavi, 01 BP 918 Cotonou, Benin.

2. Laboratory of Nutrition and Food Sciences, Faculty of Agronomy, University of Parakou, BP 2760, AbomeyCalavi, Benin.

3. Department of Animal Health and Production, Ecole Polytechnique d'Abomey-Calavi, University of AbomeyCalavi, 01 BP 2760, Abomey-Calavi, Republic of Benin.

\section{Manuscript Info}

\section{Manuscript History}

Received: 24 May 2019

Final Accepted: 26 June 2019

Published: July 2019

Key words:-

Diabetes mellitus, immunology, lifestyle, management, nutrition, physiopathology.

\begin{abstract}
Diabetes mellitus is a group of metabolic disorders characterized by the presence of chronic hyperglycemia accompanied by greater or lesser impairment in the metabolism of carbohydrates, lipids and proteins. Symptoms of high blood sugar include frequent urination, increased thirst, and increased hunger. If left untreated, diabetes can lead on many complications: diabetic ketoacidosis, hyperosmolar, hyperglycemic state, cardiovascular disease, stroke, chronic kidney disease, foot ulcers, eyes damage, or death. Diabetes is due to either the pancreas not producing enough insulin, or the cells of the body not responding properly to the insulin produced. There are different types of diabetes mellitus. Type 1 diabetes is characterized by autoimmune destruction of insulin producing cells in the pancreas by CD4+ and $\mathrm{CD} 8+\mathrm{T}$ cells and macrophages infiltrating the islets, usually leading to absolute insulin deficiency. Type 2 diabetes is due to a progressive insulin secretory defect on the background of insulin resistance. The two main physiopathological defects in type 2 diabetes are impaired insulin secretion through a dysfunction of the pancreatic $\beta$-cell, and impaired insulin action through insulin resistance. Gestational diabetes refers to first-onset glucose intolerance occurring during pregnancy. The pathophysiology of gestational diabetes mellitus and type 2 diabetes are similar; diabetes gestational could be a reflection of an early stage of type 2 diabetes occurring in the context of pregnancy. Prevention and treatment involve maintaining a healthy lifestyle, diet and nutrition, regular physical exercise, a normal body weight, avoiding use of tobacco and blood pressure control.
\end{abstract}

Copy Right, IJAR, 2019,. All rights reserved.

Corresponding Author:-Fagninou Nonsito Adnette.

Address:- Laboratory of Cell Biology and Physiology, Faculty of Sciences and Techniques, University of Abomey-Calavi, 01 BP 918 Cotonou, Benin. 


\section{Introduction:-}

Diabetes causes high human, social and economic costs for countries of all income levels, whatever their form. Type 1 and type 2 diabetes are both characterized in common by high blood glucose levels (hyperglycemia) that can cause serious health complications including ketoacidosis, kidney failure, heart disease, stroke, and blindness (Van Belle et al., 2011). Type 1 diabetes is a chronic autoimmune disease in which destruction or damaging of the beta-cells in the islets of Langerhans results in insulin deficiency and hyperglycemia. It is now established that clinical manifestation of Type 1 diabetes reflects the consequence of an underlying, sustained autoimmune process. For instance, autoantibodies against islet antigens are detected before the clinical onset of Type 1 diabetes. This suggests that a sequence of inciting events precedes the hyperglycemia for at least months, but most likely several years (Van Belle et al., 2011).

Gestational diabetes is defined as a glucose intolerance resulting in hyperglycaemia of variable severity with onset during pregnancy. In both developed and developing countries, obesity in childhood has increased dramatically. According to Buchanan and Xiang (2005), prenatal, perinatal and postnatal environmental factors impact childhood obesity. Several studies have found that intrauterine exposure to maternal gestational diabetes mellitus increases risk of long-term adverse outcomes in offspring, including obesity (Carrington et al., 1957; World Health Organization, 1999; American Diabetes Association, 2004).

This review aims to revisit the epidemiology, aetiology, physiopathology, pathogenesis, risk factors, nutrition and prevention of the different forms of diabetes in order to better understand its clinical presentation, immunology and outcomes.

\section{Classification}

According to the American Diabetes Association (2008), diabetes can be classified into the four following general categories:

1. Type 1 diabetes characterized by beta cell destruction caused by an autoimmune process, usually leading to absolute insulin deficiency. According to Kumar and Clark (2002), type 1 diabetes is usually characterized by the presence of antiglutamic acid decarboxylase, islet cell or insulin antibodies which identify the autoimmune processes that lead to beta cell destruction. All type1 diabetic patients may require insulin therapy to maintain normglycemia (Baynes, 2015).

2. Type 2 diabetes due to a progressive insulin secretory defect on the background of insulin resistance (American Diabetes Association, 2008). This is the most common form of diabetes mellitus and is highly associated with a family history of diabetes, older age, obesity and lack of exercise (Baynes, 2015). It is more common in women, especially women with a history of gestational diabetes.

3. Gestational diabetes mellitus (GDM): diabetes diagnosed in the second or third trimester of pregnancy that is not clearly overt diabetes. According to Baynes (2015), women who develop Type 1 diabetes mellitus during pregnancy and women with undiagnosed asymptomatic Type 2 diabetes mellitus that is discovered during pregnancy are classified with Gestational Diabetes Mellitus (GDM). The third trimester of pregnancy is reported to be the onset of the disorder in most women with GDM.

4. Specific types of diabetes due to other causes. This group includes persons with genetic defects of beta-cell function (neonatal diabetes and maturity-onset diabetes of the young); persons with diseases of the exocrine pancreas such as cystic fibrosis; and drug- or chemical-induced diabetes such as in the treatment of HIV/AIDS or after organ transplantation. This category represents less than $10 \%$ of diabetes cases (Baynes, 2015).

\section{Epidemiology}

Dramatic increases in the prevalence and incidence of diabetes have occurred in many parts of the world especially in the newly industrialised and developing countries in last decades (King et al., 1993; Mokdad et al., 2001; Steyn et al., 2004). Indeed, the majority of cases of type 2 diabetes in the future will occur in developing countries with India and China having more cases than any other country in the world.

Surveys carried out in adults aged 20-74 years in representative samples of the US population show that the prevalence differs considerably in different ethnic groups. Native American populations have prevalence rates of type 2 diabetes which are even higher than those of Hispanic and African Americans, although the prevalence does vary from one native American group to another (Mokdad et al., 2001; Steyn et al., 2004). It has been evaluated that 
around 366 million people worldwide or 8.3\% in the age group of 20-79 years had type 2 diabetes in 2011 (Wild et al., 2004; Shaw et al., 2010; Whiting et al., 2011).

Data from the Behavioural Risk Factor Surveys, carried out on representative samples of US adults, 18 years and older, indicated an increase of diagnosed diabetes between 1991 and 1999 from 4.1 to $6.0 \%$ in men, and from 5.6 to $7.6 \%$ in women, an increase of approximately $40 \%$ in less than a decade (Steyn et al., 2004). Turning to the situation in developing populations, in African Sub-Saharan countries, formerly, the disease either was absent, or was very low in occurrence. Even as late as 1987, in a rural village in Togo, West Africa, none of a large series of subjects examined had diabetes. In South Africa, diabetes was rare in rural areas; while the proportion affected was of 1.1\% in urban zones (Yen et al., 1971).

Diabetes is a therefore a real public health problem. There are more than 100 million diabetics worldwide (Papoz et al., 1994, Grimaldi 2000). The term diabetes actually covers two different diseases:

1. insulin-dependent diabetes (type 1), which occurs most often before the age of 20 and represents 10 to $15 \%$ of diabetes;

2. Non-insulin-dependent diabetes (type 2), which occurs most often after the age of 50 and accounts for 85 to $90 \%$ of diabetes.

It is non-insulin-dependent diabetes that poses a public health problem. Its prevalence increases with aging, urbanization, settlement and the development of obesity in the populations of developed countries. However, this disease does not spare the developing countries where non-insulin-dependent diabetes sometimes reaches a prevalence of 20 to $30 \%$, due to a genetic predisposition coupled with a rapid change in lifestyle: brutal urbanization, settlement and alcoholism of populations (Grimaldi 2000; Steyn et al., 2004).

Diabetes is of significant financial cost because of the high rate of degenerative complications. The cost of diabetes is estimated at 35 billion euros. To reduce this cost, the Saint Vincent Declaration adopted in 1989 by the World Health Organization (WHO), European governments and organizations of patients, recommended the good medical practices in dialectology (Grimaldi 2000; Steyn et al., 2004).

About the mortality, diabetes is associated with excess mortality mainly attributable to the vascular complications of the disease. According to Steyn et al. (2004), in Caucasian populations, much of the mortality is due to cardiovascular disease, mainly ischaemic heart disease (Roper et al., 2001; Morrish et al., 2001; Steyn et al., 2004), but in others such as Asian and American Indian populations renal disease contributes to a considerable extent (Steyn et al., 2004). In some developing nations, an important component of the mortality is due to infections (Steyn et al., 2004). Age-adjusted mortality rates among persons with diabetes are 1.5-2.5 times higher than in the general population (Roper et al., 2001; Morrish et al., 2001) with the greatest excess found in younger age groups. Life expectancy decreases among persons with type 2 diabetes (Steyn et al., 2004).

\section{Pathogenesis And Physiopathology}

There is a direct link between hyperglycemia and physiological \& behavioral responses (Steyn et al., 2004; Baynes, 2015). Type 1 diabetes is due to an autoimmune destruction of insulin secretory cells called B cells. Hyperglycemia occurs when only 10 to $20 \%$ of functional B cells remain. The autoimmune process responsible for pancreatic "insulitis" takes place over many years (5 to 10 years or more before the onset of diabetes). This autoimmune reaction occurs in a field of genetic susceptibility as a result of triggering factors and can be detected before the onset of hyperglycemia by blood assays of autoantibodies (Grimaldi, 2000).

The destruction of cell B is mainly due to islet infiltration by CD4 helper T lymphocytes and CD8 cytotoxic T lymphocytes. This process takes place without any clinical sign for several years. During this reaction, autoantibodies to certain pancreatic antigens are produced. These autoantibodies do not in themselves have a pathogenic role but are reliable markers of the course of the pathological autoimmune process (Grimaldi, 2000). These antibodies are essentially 4:

1. The anti-islet antibodies (islet cell antibody: ICA).

2. Anti-GAD antibodies (glutamate acid decarboxylase). These antibodies are directed against a ubiquitous enzyme but are expressed at the pancreatic level. Their presence reflects the existence of an autoimmune process directed against pancreatic B cells.

3. Anti-insulin autoantibodies, found mainly in children. 
4. The anti-IA2 antibody: it is an antibody directed against a membrane phosphatase of the B cells.

Although the main defect reported in Type 1 diabetes is insulin deficiency (Baynes, 2015), there is also a defect in the administration of insulin. Deficiency in insulin leads to uncontrolled lipolysis and great levels of plasma free fatty acids, which suppresses glucose metabolism in peripheral tissues (Holt, 2004; American Diabetes Association, 2010; Raju and Raju, 2010). This affects the use of glucose and insulin deficiency also reduces the expression of genes necessary for the response of target tissues to insulin (glucokinase in liver and the GLUT 4 class of glucose transporters in adipose tissue). Therefore, the major metabolic disorbers, which result from insulin deficiency in Type 1 diabetes are impaired glucose, lipid and protein metabolism (Holt, 2004; American Diabetes Association, 2010; Raju and Raju, 2010; Baynes, 2015).

In short, type 1 Diabetes is characterized by autoimmune destruction of insulin producing cells in the pancreas by CD4+ and CD8+ T cells and macrophages infiltrating the islets (Grimaldi, 2000; Steyn et al., 2004, Van Belle et al., 2011; Baynes, 2015).

In type 2 diabetes, the two main physiopathological defects are impaired insulin secretion through a dysfunction of the pancreatic $\beta$-cell, and impaired insulin action through insulin resistance (Holt, 2004; American Diabetes Association, 2010; Raju and Raju, 2010; Baynes, 2015). The $\beta$-cells increase the insulin supply and compensate for the excessive and anomalous demand, when resistance to insulin predominates (Van Belle et al., 2011; Baynes, 2015).

As for gestational diabetes, the pathophysiology of gestational diabetes mellitus and type 2 diabetes are similar, so that diabetes gestational could be a reflection of an early stage of type 2 diabetes occurring in the context of pregnancy.

According to Buchanan and Xiang (2005), pregnancy is characterized by an increase in insulin resistance, which is correlated with the progress of gestational age. The foeto-placental unit is at the origin of this insulin resistance and, although the mechanisms are not completely known, the production placenta of Tumor Necrosis Factor- $\alpha$ (TNF- $\alpha$ ), placental lactogen hormone, growth hormone and increased blood levels of cortisol and progesterone are contributing factors clearly demonstrated. To reduce this increase in insulin resistance and maintain normoglycemia in the mother, the production of insulin by the $\beta$ cells is also increase (Holt, 2004; American Diabetes Association, 2010; Raju and Raju, 2010; Baynes, 2015). The resulting changes in the metabolism of carbohydrates and lipids in the mother ensure that adequate nutrition reaches the fetus. Delivery of glucose to the fetus, mediated by the placenta, is facilitated by a $30 \%$ increase in endogenous production maternal basal glucose hepatic. The women who are unable to adapt sufficiently to these physiological changes induced by pregnancy develop gestational diabetes (Holt, 2004; Buchanan and Xiang, 2005; Dabelea, 2007; American Diabetes Association, 2010; Raju and Raju, 2010; Wu, 2013; Baynes, 2015).

According to Yessoufou (2006), irrespective to their etiology, diabetes and obesity are related to the alteration of the antioxidant status, lipid metabolism and immune status. Feeding cassava should not be recommended for diabetic patients as it aggravates the diabetic complications. Cassava may be consumed by non-diabetic subjects with sufficient protein content. Indeed, fish oil-enriched diet presents beneficial effects in diabetic animals and their macrosomic/obese offspring, as it improves antioxidant and immune status and corrects the hyperlipidaemia. As PPAR $\alpha$ being activated by n-3 polyunsaturated fatty acids (PUFA), and immunosuppressive and lipid catabolism activator, it may constitute a potential target molecule in the treatment of hyperlipidaemia and inflammatory state which characterize diabetes and obesity.

\section{Cytokine Levels In Gestational Diabetes Mellitus}

In last decade, several studies the role of the inflammatory system in the pathogenesis of Type 2 diabetes and gestational diabetes mellitus were deeply carried out (Gillman et al., 2003; Richardson and Carpenter, 2007 ; Tam et al., 2008; Commins et al., 2010; Donath and, Shoelson, 2011). Cytokines, a group of proteins that are expressed by several cell types, act as immune mediators and regulators (Commins et al., 2010). Gestational diabetes mellitus is an inflammatory condition that involves unbalanced cytokine production. Gomes et al. (2013) had reported that Cytokine TNF-A concentration was slightly higher in GDM than in control patients, although not significant. However, there are several factors that influence cytokine production during pregnancy, including gestational age, ethnicity, smoking and body mass index (American Diabetes Association, 2012; Matarese et al., 2012). 
Indeed, a predominant inflammatory profile defined by increased production of Th1 cytokines (interferon-gamma and tumor necrosis factor-alpha), are reported to compromise the normal development of the concept according to the period of pregnancy (Commins et al., 2010; Gomes et al., 2013). Matarese et al. (2012) and American Diabetes Association (2012) showed that insulin resistance is correlated with abnormal secretion of pro-inflammatory cytokines and decreased production of anti-inflammatory mediators.

\section{Complications}

The complications of GDM diabetes depend on the management moment during the pregnancy. Management after 2 months increases the risk of malformation by 5 to 6 times compared to pre-conception care. They are nonspecific for diabetes (except caudal regression syndrome) and most often lead on cardiac malformations or to neurological malformations (spina bifida, hydrocephalus, anencephaly, renal malformations). The consequences of these malformations are spontaneous miscarriages or neonatal mortalities. Management of gestational diabetes mellitus (GDM) in the second trimester can lead on hyperglycemia with excess of amino acids and free fatty acids, fetal hyperinsulinism, fetal hyperanabolism, macrosomia developed; tissue hypoxia, delayed pulmonary maturation (directly related to hyperinsulinemia), septal cardiac hypertrophy. During delivery, complications such as fetal trauma secondary to macrosomia, severe hypoglycaemia of the child, hypocalcemia (sudden deficiency of maternal hyperanabolism), hyperbilirubinemia / polycythemia (secondary to hypoxia), transient respiratory distress due to amniotic fluid resorption delay, hyaline limb disease are reported (Grimaldi, 2000; Steyn et al., 2004, Van Belle et al., 2011; Baynes, 2015). Furthermore, in mother, gestational diabetes is associated with increased risk preeclampsia and caesarean section. For the longer term, women who have gestational diabetes have a risk of developing recurrence of gestational diabetes between $30-84 \%$ of a subsequent pregnancy and have a risk multiplied by 7 to develop type 2 diabetes, a heightened risk by 2 to 5 to develop a metabolic syndrome and a risk multiplied by 1.7 to develop later a heart disease. Type 2 diabetes can appear in postpartum ( 5 to $14 \%$ of cases) or later (Holt, 2004; Buchanan and Xiang, 2005; American Diabetes Association, 2010; Raju and Raju, 2010; Baynes, 2015).

Maternal hyperglycemia during pregnancy is correlated with increased risk of obesity in childhood. Some studies have also demonstrated that a hyperglycemic intrauterine environment could play a role in the development of diabetes type 2 and metabolic syndrome in adulthood (Grimaldi, 2000; Steyn et al., 2004, Van Belle et al., 2011; Baynes, 2015).

\section{Risk Factors}

The risk factors of diabetes can be classified into two categories: irreversible risk factors and modifiable risk factors. Irreversible risk factors include race/ethnicity, familial aggregation, genetic factors, age and gender. Modifiable risk factors are: obesity, physical inactivity, quantity and quality of fat consumed, quantity and quality of carbohydrates consumed, micronutrients (vitamine E, magnesium, chromium), alcohol intake, intrauterine environment and environmental factors (Steyn et al., 2004).

Indeed, the prevalence of type 2 varies considerably among populations of different ethnic origins living in apparently similar environments. The empirical risk of having type 2 diabetes is increased 2 to 6 -fold if a parent or sibling has the disease (King et al., 1993; Mokdad et al., 2001; Steyn et al., 2004). The prevalence and incidence of type 2 diabetes vary to some extent between the sexes from one population to another, but these differences are relatively small and appear to be accounted for by differences in other risk factors such as obesity and physical activity (Steyn et al., 2004). The prevalence of type 2 diabetes increases with age although the patterns of incidence vary considerably. Obesity, appreciated by the body weight index increases the risk of diabetes. The abdominal, subcutaneous and even more visceral distribution of fats are also some risk factors. A sedentary lifestyle increases the risk of diabetes by 2 . Genetically, insulin resistance could be explained by an increase in fast-growing muscle fibers that are more insulin-resistant than slow-twitch fibers. Indeed, so-called type 1 slow-twitch fibers are richly vascularized with oxidative metabolism, and are very sensitive to insulin (King et al., 1993; Mokdad et al., 2001; Steyn et al., 2004). They are solicited by endurance efforts and their number is increased among trained athletes. In contrast, so-called type 2 fast-twitch fibers are insulin-resistant. The topographic distribution of adipose tissue and the typological variation of muscle tissue depend on hormonal and environmental factors: stress, alcohol, and smoking favor the android topography of fats, whereas the sedentary lifestyle and aging lead to an increase in muscle fibers (Grimaldi 2000; Kumar and Clark, 2002; Steyn et al., 2004; Van Belle et al., 2011; Baynes, 2015). Furthermore; the elderly person accumulates several factors of insulin resistance. Essential arterial hypertension, increased triglycerides and lower HDL cholesterol appear as consequences of insulin resistance, which would account for the frequency of their association with diabetes of type 2 (Grimaldi, 2000). 
About fat consumption, both quantity and quality of dietary fat are reported to modify glucose tolerance and insulin sensitivity (Steyn et al., 2004; Van Belle et al., 2011; Baynes, 2015). A high fat content in the diet leads on deterioration of glucose tolerance by several mechanisms including decreased binding of insulin to its receptors, impaired glucose transport, reduced proportion of glycogen synthase and accumulation of stored triglycerides in skeletal muscle (Van Belle et al., 2011; Baynes, 2015). The fatty acid composition of the diet, in turn, affects tissue phospholipid composition, which may relate to insulin action by altering membrane fluidity and insulin signaling (Grimaldi 2000; Kumar and Clark, 2002; Steyn et al., 2004; Van Belle et al., 2011; Baynes, 2015).

Environmental factors are likely to trigger the autoimmune process. The role of viruses in the pathogenesis of type 1 diabetes is suspected but not demonstrated. The high prevalence of type 1 diabetes (about 20\%) in case of congenital rubella or the presence of coxsackie B4 virus isolated in the pancreas of a child who died during an inaugural ketoacidosis (Grimaldi, 2000) justify this hypothesis. Some viruses may have a common antigen with B-cell proteins (coxsakie virus or cytomegalovirus). The viral infection could be responsible for the secretion of cytokines, in particular interferon, which promotes the development of the autoimmune reaction at the pancreatic level by various mechanisms.

Among the classic risk factors, those with the more impact are the personal history of diabetes gestational, age, maternal age and obesity (Grimaldi 2000; Kumar and Clark, 2002; Steyn et al., 2004; Van Belle et al., 2011; Baynes, 2015). Ethnic origin and the family history of type 2 diabetes have a significant but more moderate influence. Many unconventional factors were characterized during in recent years, whether they are physiological (low birth weight, small maternal height) or pathological (insulin resistance, ovarian syndrome polycystic). The multiplicity of risk factors and their interactions makes it difficult to predict risk individual.

Several other risk factors have been related to the development of diabetes. These include several inflammatory markers (interleukin-6, C-reactive protein, other cytokines and acute phase reactants) and variation in levels of sex hormones.

\section{Management Of Diabetes, Nutrition And Prevention}

Losing weight and increasing physical activity can prevent or delay the development of type 2 diabetes (T2D). Treatment goals focus on controlling blood glucose, blood pressure, and lipids with emphasis on low-density lipoprotein cholesterol to prevent micro- and macrovascular complications. Nutritional management of diabetes, also known as diabetes medical nutrition therapy, is based on individual assessment to develop a personalized tailored evidence-based treatment plan. The distribution of macronutrient and dietary pattern may vary based on a number of factors, including matching insulin to lifestyle in type 1 diabetes and weight loss in T2D (Wylie-Rosett and Delahanty, 2017).

Before insulin was developed as a therapy, reducing carbohydrate intake was the main treatment for diabetes. Carbohydrate restriction for the treatment of type 2 diabetes has been an area of intense interest because, of all the macronutrients, carbohydrates have the greatest effect on blood glucose and insulin levels.

About foods to avoid, consensus exists on reducing or avoiding the intake of processed red meats, refined grains and sugars (especially sugar sweetened drinks) both for prevention and management of type 2 diabetes, again with some cautions. Firstly, for unprocessed red meat, the evidence of possible harm because of the development of type 2 diabetes is less consistent and of a smaller magnitude. Secondly, evidence is increasing on the relevance of carbohydrate quality: that is that whole grains and fiber are better choices than refined grains and that fibre intake should be at least as high in people with type 2 diabetes as recommended for the general population, that diets that have a higher glycaemic index and load are associated with an increased risk of type 2 diabetes, and that there is a modest glycaemic benefit in replacing foods with higher glycaemic load with foods with low glycaemic load (Westman et al., 2006).

The management of diabetes can be based on the promotion and evaluation of healthy lifestyle projects which focus on the prevention and early treatment of overweight and obesity; the consumption of a nutrient-dense diet poor in fat, particularly saturated fat, and free sugars and high in non-starch polysaccharides; an active lifestyle which includes regular physical activity of at least 1 hour per day, and vigorous activity, which is required to reduce the risk of developing type 2 diabetes; cessation of alcohol intake and cigarette smoking. 
Indeed, weight loss achieved by an increase in physical activity and dietary change including reduction in total and saturated fat and increased dietary fiber can reduce the incidence of diabetes. Other studies, shows that reduction of early feeding may also play a role in subsequent reduction of type 2 diabetes in later life.

Therefore, dietetics, glycemic self-monitoring, insulin therapy if necessary are a number of specific methods of gestational diabetes management. These specific methods of gestational diabetes management reduce severe perinatal complications, fetal macrosomia and preeclampsia, by comparison therapeutic abstention, without increasing the risk of caesarean section.

As for prevention, physical activity, the use of food supplements such as probiotics, fish oil, or metformin are recommended to prevent the appearance of diabetes, with the aim of reducing complications and the cost of health.

According to Forouhi et al. (2018), the following suggestions must be taken into account in the prevention of type 2 diabetes:

1. Dietary approaches for the prevention and management of type 2 diabetes is recommended although uncertainties remain. These approaches may be associated with weight management as a cornerstone of metabolic health, and diet quality.

2. Low carbohydrate diets are ideal for weight management and glycaemic control

3. Dietary advice should be on foods and healthy eating patterns rather than on nutrients. Evidence supports avoiding processed foods, refined grains, processed red meats, and sugar sweetened drinks and promoting the intake of fiber, vegetables, and yoghurt. Dietary advice should be individually tailored and take into account personal, cultural, and social factors.

\section{Future Research Directions On Diabetes}

Important studies are needed to dissect the various mechanisms underlying diabetes, especially gestational diabetes mellitus and its evolution after pregnancy. Large studies of biochemical, immunological and genetic bases of the metabolic memory associated with gestational diabetes inducing fetal macrosomia, obesity and diabetes in adult offspring are necessary to more accurately define the clinical characteristics of women who need such screening as part of routine GDM management. Furthermore, genetic studies are needed to identify persons whose $\beta$ cells will tolerate insulin resistance poorly, as well as persons who develop poor insulin secretion for reasons unrelated to insulin resistance.

\section{Conclusion:-}

The review of bibliographies suggests that diabetes is a metabolic defects characterized by the presence of chronic hyperglycemia either immune-mediated (Type 1 diabetes), insulin resistance (Type 2), gestational or others such as environment, genetic defects, infections, and certain drugs. Gestational diabetes mellitus is an inflammatory condition that involves unbalanced cytokine production. Physical activities, use of food supplements such as probiotics, fish oil, or metformin are recommended to prevent the appearance of diabetes. Pregnant women who have hyperglycemia can control their blood sugar levels through healthy nutrition, light exercise, and blood glucose monitoring. Overall, lifestyle and pharmacological interventions can reduce incident diabetes and delay its progression.

\section{References:-}

1. American Diabetes Association (2008): Diagnosis and Classification of Diabetes Mellitus, Diabetes Care 2008 31: 55-60.

2. American Diabetes Association (2010): Diagnosis and classification of diabetes mellitus. Diabetes Care 33 Suppl 1: 62-69.

3. American Diabetes Association (2004): Diagnosis and classification of diabetes mellitus. Diabetes Care; 27(Suppl. 1): 5-10.

4. American Diabetes Association (2012): Diagnosis and classification of diabetes mellitus. Diabetes Care 2012; 35 Suppl 1:64-S71.

5. Baynes, H.W. (2015): Classification, Pathophysiology, Diagnosis and Management of Diabetes Mellitus. J Diabetes Metab 6: 541. doi:10.4172/2155-6156.1000541.

6. Buchanan, T.A. and Xiang, A.H. (2005): Gestational diabetes mellitus. J Clin Invest. Mar 1; 115(3): 485-491.

7. Carrington, E.R., Shuman, C.R., Reardon, H.S. (1957): Evaluation of the prediabetic state during pregnancy. Obstet. Gynecol. 9: 664-669. 
8. Commins, S.P., Borish, L., Steinke, J.W. (2010): Immunologic messenger molecules: cytokines, interferons, and chemokines. J Allergy Clin Immunol. 125:53-72.

9. Dabelea, D. (2007): The predisposition to obesity and diabetes in offspring of diabetic mothers. Diabetes care 30:169-174.

10. Donath, M.Y., Shoelson, S.E. (2011): Type 2 diabetes as an inflammatory disease. Nat Rev Immunol 2011; 11:98-107.

11. Forouhi, G.N., Misra, A., Mohan, V., Taylor, R., Yancy, W. (2018): Dietary and nutritional approaches for prevention and management of type 2 diabetes. Science and Politics of Nutrition, BMJ 361 doi: https://doi.org/10.1136/bmj.k2234 .

12. Gillman, M.W., Rifas-Shiman, S, Berkey, C.S., Field, A.E., Colditz, G.A. (2003): Maternal gestational diabetes, birth weight, and adolescent obesity. Pediatrics. 111: 221-226.

13. Gomes, C.P., Torloni, M.R., Gueuvoghlanian-Silva, B.Y., Alexandre, S.M., Mattar, R., Daher, S. (2013): Cytokine levels in gestational diabetes mellitus: a systematic review of the literature. Am J Reprod Immunol. 69: 545-557.

14. Holt, R.I. (2004): Diagnosis, epidemiology and pathogenesis of diabetes mellitus: an update for psychiatrists. Br J Psychiatry Suppl 47: 55-63.

15. King, H, Rewers, M. (1993): Global estimates for prevalence of diabetes mellitus and impaired glucose tolerance in adults. WHO Ad Hoc Diabetes Reporting Group. Diabetes Care 1993; 16: 157-77.

16. Kumar, P.J., Clark, M. (2002): Textbook of Clinical Medicine. Pub: Saunders, London, UK. 1099-1121.

17. Matarese, G., Procaccini, C., de Rosa, V. (2012): At the crossroad of T cells, adipose tissue, and diabetes. Immunol Rev 249:116-134.

18. Mokdad, A.H., Bowman, B.A., Ford, E.S., Vinicor, F., Marks, J.S., Koplan, J.P. (2001): The continuing epidemics of obesity and diabetes in the United States. Journal of the American Medical Association; 286: 1195-200.

19. Morrish, N.J., Wang, S.L., Stevens, L.K., Fuller, J.H., Keen, H. (2001): Mortality and causes of death in the WHO multinational study of vascular disease in diabetes. Diabetologia 44: 14-21.

20. Raju, S.M., Raju, B. (2010): Illustrated medical biochemistry. 2nd Edition. JaypeeBrothers Medical Publishers ltd, New Delhi, India.

21. Regnault, N., Gillman, M.W., Rifas-Shiman, S.L., Eggleston, E., Oken, E. (2013): Sex-specific associations of gestational glucose tolerance with childhood body composition. Diabetes care 36:3045-3053.

22. Richardson, A.C., Carpenter, M.W. (2007): Inflammatory mediators in gestational diabetes mellitus. Obstet Gynecol Clin North Am; 34: 213-224.

23. Roper, N.A., Bilous, R.W., Kelly, W.F., Unwin, N.C., Connolly, V.M. (2001): Excess mortality in a population with diabetes and the impact of material deprivation: longitudinal, population based study. British Medical Journal; 322: 1389-93.

24. Shaw, J.E., Sicree, R.A., Zimmet, P.Z. (2010): Global estimates of the prevalence of diabetes for 2010 and 2030. Diabetes Res Clin Pract; 87: 4-14.

25. Tam, W.H., Ma, R.C., Yang, X. (2008): Glucose intolerance and cardiometabolic risk in children exposed to maternal gestational diabetes mellitus in utero. Pediatrics; 122: 1229-1234.

26. Van Belle, T.L., Coppieters, K.T., and Von Herrath, M.G. (2011): Type 1 Diabetes: Etiology, Immunology, and Therapeutic Strategies. Physiological ReviewsVol. 91, No. 1. https://doi.org/10.1152/physrev.00003.2010.

27. van Belle, T.L., Coppieters, K.T., von Herrath, M.G. (2011): Type 1 diabetes: etiology, immunology, and therapeutic strategies. Physiol Rev; 91(1):79-118. doi: 10.1152/physrev.00003.2010.

28. Westman, E.C., Yancy, W.S., Humphreys, M. (2006): Dietary treatment of diabetes mellitus in the pre-insulin era (1914-1922). Perspect Biol Med; 49:77-83.

29. Whiting, D.R., Guariguata, L., Weil, C., Shaw, J. (2011): Global estimates of the prevalence of diabetes for 2011 and 2030. Diabetes Res Clin Pract. 94: 311-21.

30. Wild, S., Roglic, G., Green, A., Sicree, R., King, H. (2004): Global prevalence of diabetes: Estimates for the year 2000 and projections for 2030. Diabetes Care; 27: 1047-53.

31. World Health Organization (1999): Definition, diagnosis and classification of diabetes mellitus and its complications. World Health Organization; Geneva: 1999.

32. Wu, J.F. (2013): Childhood obesity: a growing global health hazard extending to adulthood. Pediatrics and neonatology; 54:71-72.

33. Wylie-Rosett, J., Delahanty, L.M. (2017): Nutrition in the Prevention and Treatment of Disease, (Fourth Edition), Pages 691-707. https://doi.org/10.1016/B978-0-12-802928-2.00031-X 
34. Yen, S.C.C., Tsai, C.C., Vela, P. (1971): Gestational diabetogenesis: quantitative analysis of glucose-insulin interrelationship between normal pregnancy and pregnancy with gestational diabetes. Am. J. Obstet. Gynecol. 111: 792-800.

35. Yessoufou, A, Ategbo, J.M., Girard, A., Prost, J., Dramane, K.L., Moutairou, K., Hichami, A., Khan, N.A. (2006): Cassava enriched diet is not diabetogenic rather it aggravates diabetes in rats. Fundam Clin Pharmacol 20: 579-586. 